\title{
Identifying placebo responders and predictors of response in osteoarthritis: a protocol for individual patient data meta-analysis
}

Yu Fu ${ }^{1 \dagger}$, Monica S. M. Persson ${ }^{2,3+}$, Archan Bhattacharya ${ }^{4,5}$, Siew-Li Goh ${ }^{2,4,6}$, Joanne Stocks ${ }^{2,3}$, Marienke van Middelkoop ${ }^{7}$, Sita M. A. Bierma-Zeinstra ${ }^{7}$, David Walsh ${ }^{2,3}$, Michael Doherty 2,3 and Weiya Zhang ${ }^{2,3^{*}}$

\begin{abstract}
Background: The management of osteoarthritis $(\mathrm{OA})$ is unsatisfactory, as most treatments are not clinically effective over placebo and most drugs have considerable side effects. On average, $75 \%$ of the analgesic effect from OA treatments in clinical trials can be attributed to a placebo response, and this response varies greatly from patient to patient. This individual patient data (IPD) meta-analysis aims to identify placebo responders and the potential determinants of the placebo response in OA.

Methods: This study is undertaken in conjunction with the OA Trial Bank, an ongoing international consortium aiming to collect IPD from randomised controlled trials (RCTs) for all treatments of OA. RCTs for each treatment of OA have been systematically searched for, and authors of the relevant trials have been contacted to request the IPD. We will use the IPD of placebo-controlled RCTs held by the OA Trial Bank for this project. The IPD in placebo groups will be used to investigate the placebo response according to the minimum clinically important difference (MCID) threshold (e.g. $20 \%$ pain reduction). Responders to placebo will be compared with non-responders to identify predictors of response. The quality of the trials will be assessed and potential determinants will be examined using multilevel logistic regression analyses.
\end{abstract}

Discussion: This study explores the varying magnitude of the placebo response and the proportion of participants that experience a clinically important placebo effect in OA RCTs. Potential determinants of the placebo response will also be investigated. These determinants may be useful for future studies as it may allow participants to be stratified into groups based on their likely response to placebo. The results of this study may also be useful for pharmaceutical companies, who could improve the design of their studies in order to separate the specific treatment from the non-specific contextual (i.e. placebo) effects.

Systematic review registration: PROSPERO CRD42016033212

Keywords: Osteoarthritis, Placebo response, Individual patient data meta-analysis

\footnotetext{
* Correspondence: weiya.zhang@nottingham.ac.uk

${ }^{\dagger}$ Equal contributors

${ }^{2}$ Academic Rheumatology, University of Nottingham, Clinical Sciences

Building, City Hospital, Nottingham NG5 1PB, UK

${ }^{3}$ Arthritis Research UK Pain Centre, Nottingham, UK

Full list of author information is available at the end of the article
} 


\section{Background}

Osteoarthritis (OA) is the most common form of arthritis worldwide [1]. People with OA often experience pain alongside impaired mobility and participation, resulting in reduced quality of life [2]. There is no cure for OA, and available treatments aim to optimise pain management using both pharmacological and non-pharmacologic modalities [3]. Of 51 currently available treatment options for the symptomatic management of OA, most do not achieve a minimum clinically important difference (MCID) (i.e. an effect size $\geq 0.5$ ) over placebo $[4,5]$. Furthermore, most pharmacological treatments have a number of side effects.

In contrast, research and clinical evidence show that placebo or sham interventions appear to be effective for symptom control in OA [6] and in other conditions including pain [7], depression [8], chronic fatigue syndrome [9], asthma [10], hypertension [11], and Parkinson's disease [12]. Previous studies suggest that the benefits of placebo interventions are mainly for patient symptoms and distress, which are considered to be the principal treatment targets in people with OA [6]. A recent meta-analysis showed that, on average, $75 \%$ of the analgesic effect from OA treatments can be attributed to placebo response and this response varies greatly from patient to patient [13].

However, debate continues as to whether the estimation of placebo response is adequate and accurate. This is largely due to the fact that the placebo response is measured as the overall change from baseline in the placebo group [14]. It is difficult to differentiate this from the regression to the mean and the natural disease fluctuation unless a notreatment group or waiting-list group is included in the trial [15-17]. However, Vase et al. [18] argued that the placebo response is robust and enhances the treatment effect when conditions are amenable to placebo, when placebo is given as an analgesic or when participants are properly blinded [19]. Furthermore, a systematic review [14] involving 198 randomised controlled trials (RCTs) also suggested that placebo is effective at relieving pain and improving function and stiffness. Its effect size ( $\mathrm{ES}=0.51,95 \% \mathrm{CI} 0.46$ to 0.55 ) was found to be significantly greater than that observed in untreated, observation-only controls $(\mathrm{ES}=0.03,95 \% \mathrm{CI}$ -0.13 to 0.18 ).

When studying the placebo response, it is essential to employ correct and robust methodology. Given the fact that predictors of placebo response may be person-specific (e.g. age and gender) or study-specific (e.g. sample size and allocation concealment), we need to consider both person and study level characteristics. Individual patient data (IPD) meta-analysis permits both individual patient level and study level predictors of placebo response to be taken into account. Moreover, it allows the researchers to use the existing datasets from RCTs instead of undertaking a large and expensive trial. This approach has been advocated by both the European League Against Rheumatism (EULAR) and the Osteoarthritis Research Society International (OARSI), who have further endorsed an international collaboration for the development of OA Trial Bank [20]. The OA Trial Bank is an ongoing international consortium aiming to collect IPD from existing RCTs for all treatments in OA. Currently, the Bank has completed an IPD analysis for intra-articular (IA) glucocorticoids [21] and is collecting the IPD for glucosamine, topical non-steroidal antiinflammatory drugs (NSAIDs), and topical capsaicin in OA. This provides a representative sample of the treatments in OA, spanning across various modes of delivery including tablets, injections, and topical formulations, for this project. The aim of this review is to identify placebo responders and predictors of response in OA.

\section{Methods/design}

An IPD meta-analysis of RCTs will be undertaken to identify placebo responders and to investigate the predictors of placebo response in participants with OA. In order to increase transparency, the PRISMA-P checklist [22] was referred to as Additional file 1.

\section{Study selection}

RCTs of IA glucocorticoids, glucosamine, topical NSAIDs, and topical capsaicin in OA have been systematically searched. Placebo-controlled RCTs collected in our IA glucocorticoids [21], glucosamine, topical NSAID, and topical capsaicin (PROSPERO registration number: CRD42016035254) [23] studies will be included in this IPD analysis.

\section{Type of studies}

All placebo-controlled RCTs identified for the above four treatments, including crossover trials, will be included. Trials for other types of arthritis, such as rheumatoid arthritis, will be excluded. Studies of non-clinical outcomes, including biomarkers and animal models, will be excluded. No language restrictions will be applied to this review.

\section{Participants}

Participants in the above RCTs diagnosed with OA, as defined by the criteria endorsed by the American College of Rheumatology [24, 25] or by the use of clearly defined radiographic and clinical criteria, will be included.

\section{Types of interventions}

This review focuses on the interventions used in the control arms of trials collected in the OA Trial Bank. This includes participants receiving placebo interventions (regardless of the mode of delivery and dose), participants on waiting lists, and participants under standard care. 


\section{Types of baseline assessments}

As a minimum, studies will need to record pain, age, and gender at baseline. Other clinical characteristics, including physical function, stiffness, and pain elsewhere, will be included if available.

\section{Types of outcomes}

The minimum criterion for inclusion is reporting of pain. The primary outcome measure for investigating placebo response is pain reduction at 4 weeks of follow-up. Secondary analyses will include pain reduction at other durations of follow-up, as well as functional impairment and patient global assessment, as recommended by the OMERACTOARSI Initiative [26, 27].

\section{Identification of eligible studies}

Trials eligible for inclusion in this analysis will be identified from the IPD collected in the OA Trial Bank for IA glucocorticoids [21], glucosamine, topical NSAIDs, and topical capsaicin reviews (PROSPERO registration number: CRD42016035254) [23]. Each treatment was systematically reviewed (MM for IA steroid, JR for glucosamine, and MSMP for topical NSAIDs and capsaicin). The process was similar for all reviews and is summarised below.

A search strategy was developed by the lead reviewer and refined in consultation with team members, including physicians, librarians, and researchers experienced in conducting systematic reviews. A systematic literature search was then undertaken by the lead reviewer. Databases searched include Cochrane library, MEDLINE, Embase, AMED, Web of Science, Scopus, Pedro, and CINAHL [21]. The identified studies were exported to Endnote, where eligibility for inclusion was assessed by two independent reviewers. If no consensus was reached, a third researcher was consulted.

In addition to the systematic literature search, efforts were made to identify unpublished trials by contacting pharmaceutical suppliers and reviewing the British National Formulary, the electronic Medicines Compendium, and Clinicaltrials.gov.

\section{Data collection and transfer}

All corresponding authors of eligible trials have been invited to collaborate by MM, JR, and MSMP. Corresponding authors that are interested in collaboration have been asked to sign a data delivery agreement that has been drafted on behalf of the OA Trial Bank. The agreement includes items on input data, obligations, ownership of data, terms, authorship, and publications. Data has been contributed to the OA Trial Bank by the corresponding author and is stored on a secure server. Data has been accepted in any format and is kept anonymised and confidential.

The corresponding authors of placebo-controlled trials stored in the OA Trial Bank will be contacted. The authors will be asked to sign a further data transfer agreement allowing the use of their data for this review before the transfer of the data to The University of Nottingham for analysis.

\section{Risk assessment}

The quality of the included trials will be independently assessed by two reviewers using a modified version of the Risk of Bias tool recommended by the Cochrane Collaboration [28]. Whenever there is a disagreement, a third independent researcher will be consulted until a consensus is reached. The modified Risk of Bias Tool is composed of nine questions that measure each of the domains included in the tool (Table 1). For each domain, studies will be rated as a "low risk", "high risk", or "unclear risk" of bias. Clear criteria for the ratings will be followed, and a justification for the rating will be required. Studies will then be categorised as "low risk", "high risk", or "unclear risk". A study with low risk is defined as fulfilling at least five of the criteria questions.

All trials, irrespective of quality, will be included in the primary data analyses. Subsequently, a subgroup analysis will be undertaken to examine whether the quality of the study influences the outcomes, for example, whether higher quality studies have a greater placebo response.

Table 1 Modified risk of bias assessment

\begin{tabular}{|c|c|c|c|c|}
\hline & Yes & No & Unclear & Comments \\
\hline 1. Was the randomisation procedure adequate? & $\square$ & $\square$ & $\square$ & \\
\hline 2. Was the treatment allocation adequately concealed? & $\square$ & $\square$ & $\square$ & \\
\hline 3. Were participants blinded to the intervention? & $\square$ & $\square$ & $\square$ & \\
\hline 4. Were physicians blinded to the intervention? & $\square$ & $\square$ & $\square$ & \\
\hline 5. Were outcome assessors blinded to the intervention? & $\square$ & $\square$ & $\square$ & \\
\hline 6. Incomplete outcome data: Is the attrition rate $<15 \%$ ? & $\square$ & $\square$ & $\square$ & \\
\hline 7. Are all pre-specified outcomes of interest reported in the pre-specified way? & $\square$ & $\square$ & $\square$ & \\
\hline 8. Was intention-to-treat analysis used? & $\square$ & $\square$ & $\square$ & \\
\hline 9. Were the treatment and control group similar at baseline? & $\square$ & $\square$ & $\square$ & \\
\hline
\end{tabular}




\section{Data extraction}

Data retrieved from the OA Trial Bank will include characteristics relating to the participant (age, gender, body mass index (BMI)); disease (radiographic information, signs of inflammation, muscle strength, duration of complaints, pain severity, type of pain, central sensitization, psychological assessments); placebo (topical/oral/injection, dose); trial (sample size, setting, allocation concealment, risk of bias); and outcome measures of interest (pain, function, patient global assessment, quality of life). All randomised participants with a database record will be entered into a pooled database, and all individual trials will be assigned an individual trial number.

\section{Outcomes}

The primary dichotomous outcome for all analyses will be clinically important pain relief (present/absent) at 4 weeks after treatment. This is assessed using the visual analogue scale (VAS) pain score from 0 (no pain) to $100 \mathrm{~mm}$ (worst pain ever).

Clinically important pain relief will be defined as a $20 \%$ or more reduction in VAS score from baseline within the placebo group. This threshold was chosen as it is the most commonly used threshold for the minimum clinically important improvement of relative change [29]. It has been suggested for pain and function for rheumatic diseases, including $\mathrm{OA}$, and relies on the assumption that the waiting-list or observational group has no pain reduction [14]. In trials with a waiting-list group, significant pain relief is defined as a reduction in pain $12.5 \%$ greater than that observed in the waiting-list group [30]. VAS pain score (continuous variable) will be used for secondary analyses.

If the VAS pain score has not been measured at 4 weeks, the time-point closest will be used instead. Pain scores at all other time points will be considered in the secondary analyses.

Where possible, the VAS pain score will be used for analysis. If unavailable, the WOMAC pain score will be used instead, followed by other Likert scores converted into a VAS 0-100 scale [21]. Other outcomes such as function (standardised to a 0-100 scale), global assessment, and quality of life will also be considered if available [26].

\section{Data analyses}

Descriptive analyses will be performed to present the characteristics of each individual trial and the study participants. Means and standard deviations will be used to describe normally distributed continuous data whilst medians and interquartile ranges will be used for data that are not normally distributed. Categorical data will be described using frequencies and percentages. Statistical analyses will be performed using the statistical programme Stata SE 14 (StataCorp, College Station, TX).
Any missing data will be assumed to be missing at random (MAR); therefore, observed participant characteristics will be used to impute missing data using multiple imputation [31, 32]. Missing data will be imputed within each original study prior to IPD being pooled. To test the validity of the imputation, a sensitivity analysis will be carried out to compare the results from the complete dataset with those from the imputed dataset.

A one-step approach will be used as it has been demonstrated to have greater power and flexibility over the two-step approach [33]. A multilevel logistic regression model will be fitted, taking into account the hierarchical nature of the data. Significant pain relief will act as the dependent variable and will be used to estimate the magnitude of the placebo response. Random intercepts may be introduced in the model to account for the patients nested within each trial.

All characteristics listed in the section "Data Extraction" (participant; disease-specific; placebo-specific; trial) will be considered potential predictors. These have been chosen as the current literature suggests that they are related to pain or response to therapy in OA. Participant-related information, such as age, gender, and BMI will be used at the patient level. Trial characteristics will be used as covariates at the study level. In the secondary analysis, a time-point level will be introduced for time points other than 4 weeks.

According to the availability of a waiting-list/no-treatment control, two models will be developed. Where a waiting list is unavailable, a multilevel logistic regression model will be built. The dependent variable of the model will be placebo response (yes/no), and independent variables will include all potential predictors. In terms of the modelling strategy, we will first include all potential predictors in the model. We will then develop a model only including significant predictors $(p \leq 0.05)$. Finally, we will refine the model by re-introducing the previously excluded non-significant predictors, one by one, to examine their significance and influence on the model.

Where a waiting list is available, predictors will be identified by assessing the treatment-covariate interaction in the multilevel regression model. For this model, the dependent variable will still be the placebo response (yes/no). However, the independent variables will include both the treatment (placebo yes/no) and predictors. We will develop a model using one predictor at a time, the treatment variable (i.e. placebo yes/no) and the treatment-predictor interaction term. Other predictors will be adjusted for as covariates in the model [32, 33]. A significant predictor will be identified when the treatment-predictor interaction term is statistically significant $(p \leq 0.05)$ in the model. The overall estimate of the effect of the predictor, with a $95 \%$ confidence interval and $p$ value, will be presented. 
In addition, secondary analyses will be conducted for VAS pain scores (continuous) using multilevel linear regression models. These will be conducted as detailed above, but the dependent variable will be replaced by VAS score and ANCOVA (analysis of covariance) will be used instead of the logistic regression.

The heterogeneity between trials will be tested using the $I^{2}$ value [34] and will be presented along with the $95 \%$ confidence interval around it [35].

The potential for publication bias and small study effects will be examined using a contour-enhanced funnel plot and a statistical test for asymmetry [36, 37]. A $p$ value less than 0.10 will be taken to indicate statistical evidence of asymmetry.

\section{Discussion}

This review aims to provide evidence of how the placebo effect produces a clinically important improvement in patient-centred outcomes in RCTs of OA. Potential determinants of the placebo response in people with OA will be investigated, which may be useful for future studies. Defining the patient, disease, treatment, and delivery of carespecific factors that influences the placebo response may allow stratification of patients who are most likely to improve with placebo. As placebo effect is an integral part of the treatment effect 13, the results of this study will be useful to enhance the overall treatment effect. This study will also help refine the effect size of placebo interventions for pain reduction in OA, as current evidence has not allowed a consensus on the minimum perceptible clinical improvement for placebo interventions in OA $[5,29,38]$.

Limitations of this review include the fact that the placebo responders are measured by the overall difference between baseline and endpoint, rather than the difference in effect between placebo and non-treatment groups. Future studies may be needed to examine the magnitude of the placebo response by comparing changes observed in participants in placebo groups with those not receiving treatment. As is often a limitation of IPD meta-analyses, it is possible that original trial authors may not be willing to collaborate or may not have access to the raw datasets required $[39,40]$. Consequently, we may be underpowered and miss some significant predictors. However, several approaches for overcoming these challenges will be implemented when collecting data for the OA Trial Bank. For example, attempts will be made to obtain IPD from trials in the grey literature as well as directly from pharmaceutical companies. We will also facilitate the process of data collection for original authors by accepting the data in any format $[40,41]$. Finally, conducting this review within a collaborative group like the OA Trial Bank allows pooling of resources which aims to facilitate the process [39].

The results of this study may have implications relevant to both clinical practice and pharmaceutical companies.
Clinicians will be made aware of the importance of the placebo response and its determinants. This may enable them to develop individualised treatment plans for patients that maximise the overall treatment effect, thereby improving symptoms and increasing patients' satisfaction. The results of this study may also be of interest to pharmaceutical companies who could recruit participants who are likely to have a low placebo response in their trials. This would increase the likelihood of observing a benefit for their drug over and above placebo.

\section{Additional file}

Additional file 1: PRISMA-P (Preferred Reporting Items for Systematic review and Meta-Analysis Protocols) 2015 checklist: recommended items to address in a systematic review protocol*. (DOC 895 kb)

\section{Abbreviations}

AMED: The Allied and Complementary Medicine Database; ANCOVA: Analysis of covariance; BMI: Body mass index; CINAHL: Cumulative Index to Nursing and Allied Health Literature; ES: Effect size; EULAR: European League Against Rheumatism; IA: Intra-articular; IPD: Individual patient data; MAR: Missing at random; MCID: Minimum clinically important difference; NSAID: Non-steroidal anti-inflammatory drug; OA: Osteoarthritis; OARSI: Osteoarthritis Research Society International; OMERACT: Outcome Measures in Rheumatology; PRISMA-P: Preferred Reporting Items for Systematic review and Meta-Analysis Protocols; PROSPERO: International Prospective Register of Systematic Reviews; RCTs: Randomised controlled trials; VAS: Visual analogue scale; WOMAC: Western Ontario and McMaster Universities Pain Scale

\section{Acknowledgements}

The authors acknowledge Jos Runhaar (JR) for his invaluable help in data collection and risk assessment of the glucosamine trials.

\section{Funding}

The placebo, topical NSAID, and capsaicin reviews are funded by the Arthritis Research UK (ARUK) Pain Centre and are hosted by the University of Nottingham. The OA Trial Bank infrastructure and reviews on IA glucocorticoids and glucosamine are funded by the Dutch Arthritis Foundation.

\section{Authors' contributions}

WZ is the guarantor. All authors were involved in planning the study design and will aid in interpreting the outcomes. All authors have read and approved the final manuscript. YF drafted the protocol and plans for the analysis. MSMP aided in the drafting and critical revision of the manuscript. JS and MSMP will contact original study authors for collaboration and will coordinate the data transfer. JS will conduct the data analyses with input from AB. MM and MSMP will aid in the data collection. MM, MSMP, and SLG will undertake the risk assessments. YF, MSMP, AB, SLG, JS, MM, SBZ, DW, $M D$, and $W Z$ reviewed, commented, and approved this protocol.

\section{Competing interests}

The authors declare that they have no competing interests.

Consent for publication

Not applicable.

Ethics approval and consent to participate

Not applicable.

\section{Author details}

${ }^{1}$ School of Healthcare, University of Leeds, Leeds, UK. ${ }^{2}$ Academic Rheumatology, University of Nottingham, Clinical Sciences Building, City Hospital, Nottingham NG5 1PB, UK. ${ }^{3}$ Arthritis Research UK Pain Centre, Nottingham, UK. ${ }^{4}$ Arthritis Research UK Centre for Sports, Exercise and Osteoarthritis, Nottingham, UK. ${ }^{5}$ Academic Orthopaedics, Trauma and Sports 
Medicine, Queens Medical Centre, University of Nottingham, Nottingham, UK. ${ }^{6}$ Sports Medicine Unit, University of Malaya, Kuala Lumpur, Malaysia. ${ }^{7}$ Department of General Practice, Erasmus MC Medical University, Rotterdam, The Netherlands.

\section{Received: 16 March 2016 Accepted: 21 October 2016} Published online: 28 October 2016

\section{References}

1. Loeser RF, et al. Osteoarthritis: a disease of the joint as an organ. Arthritis Rheum. 2012;64(6):1697-707.

2. Vitiello MV, et al. Cognitive behavioral therapy for insomnia improves sleep and decreases pain in older adults with co-morbid insomnia and osteoarthritis. J Clin Sleep Med. 2009;5(4):355.

3. Hochberg MC, et al. American College of Rheumatology 2012 recommendations for the use of nonpharmacologic and pharmacologic therapies in osteoarthritis of the hand, hip, and knee. Arthritis Care Res. 2012;64(4):465-74.

4. Zhang W, et al. OARSI recommendations for the management of hip and knee osteoarthritis, part I: critical appraisal of existing treatment guidelines and systematic review of current research evidence. Osteoarthr Cartil. 2007; 15(9):981-1000.

5. National Institute for Health and Care Excellence. Osteoarthritis care and management in adults. 2014. [cited 2015 8th December 2015]; Available from: http://www.nice.org.uk/guidance/CG177.

6. Doherty M, Dieppe P. The "placebo" response in osteoarthritis and its implications for clinical practice. Osteoarthr Cartil. 2009;17(10):1255-62.

7. Hróbjartsson A, Gøtzsche PC. Is the placebo powerless? An analysis of clinical trials comparing placebo with no treatment. N Engl J Med. 2001; 344(21):1594-602.

8. Kirsch I. Are drug and placebo effects in depression additive?. Biological psychiatry. 2000 Apr 15;47(8):733-5.

9. Cho HJ, Hotopf M, Wessely S. The placebo response in the treatment of chronic fatigue syndrome: a systematic review and meta-analysis. Psychosom Med. 2005;67(2):301-13.

10. Kemeny $M E$, et al. Placebo response in asthma: a robust and objective phenomenon. J Allergy Clin Immunol. 2007;119(6):1375-81.

11. Asmar R, Safar M, Queneau P. Evaluation of the placebo effect and reproducibility of blood pressure measurement in hypertension. Am J Hypertens. 2001;14(6): 546-52.

12. Fregni F, et al. Immediate placebo effect in Parkinson's disease - is the subjective relief accompanied by objective improvement? Eur Neurol. 2006;56(4):222-9.

13. Zou $\mathrm{K}$ et al. Examination of overall treatment effect and the proportion attributable to contextual effect in osteoarthritis: metaanalysis of randomised controlled trials. Ann Rheum Dis. 2016 Nov;75(11): 1964-1970

14. Zhang $W$, et al. The placebo effect and its determinants in osteoarthritis: meta-analysis of randomised controlled trials. Ann Rheum Dis. 2008;67(12): 1716-23.

15. Ernst E, Resch K. Concept of true and perceived placebo effects. BMJ. 1995; 311(7004):551.

16. Hróbjartsson A. What are the main methodological problems in the estimation of placebo effects? J Clin Epidemiol. 2002;55(5):430-5.

17. Hughes R, Carr A. A randomized, double-blind, placebo-controlled trial of glucosamine sulphate as an analgesic in osteoarthritis of the knee. Rheumatology. 2002;41(3):279-84.

18. Vase L, Riley JL, Price DD. A comparison of placebo effects in clinical analgesic trials versus studies of placebo analgesia. Pain. 2002;99(3):443-52.

19. Wampold BE, et al. The placebo is powerful: estimating placebo effects in medicine and psychotherapy from randomized clinical trials. J Clin Psychol. 2005;61(7):835-54

20. van Middelkoop M, et al. Individual patient data meta-analysis of trials investigating the effectiveness of intra-articular glucocorticoid injections in patients with knee or hip osteoarthritis: an OA Trial Bank protocol for a systematic review. Syst Rev. 2013;2(1):1-8.

21 Van Middelkoop M et al. The OA Trial Bank: meta-analysis of individual patient data from knee and hip osteoarthritis trials show that patients with severe pain exhibit greater benefit from intra-articular glucocorticoids. Osteoarthr Cartil. 2016 Jul;24(7):1143-52.

22. Shamseer $L$ et al. Preferred reporting items for systematic review and metaanalysis protocols (PRISMA-P) 2015: elaboration and explanation. BMJ 2015; 349:97647
23 Persson MSM, et al. Relative efficacy of topical non-steroidal anti-inflammatory drugs and topical capsaicin in osteoarthritis: protocol for an individual patient data meta-analysis. Systematic Reviews. 2016;5(1).

24 Altman $\mathrm{R}$, et al. Development of criteria for the classification and reporting of osteoarthritis: classification of osteoarthritis of the knee. Arthritis Rheum. 1986;29(8):1039-49.

25 Altman R, et al. The American College of Rheumatology criteria for the classification and reporting of osteoarthritis of the hand. Arthritis Rheum. 1990;33(11):1601-10.

26 Pham T, et al. OMERACT-OARSI Initiative: Osteoarthritis Research Society International set of responder criteria for osteoarthritis clinical trials revisited. Osteoarthr Cartil. 2004;12(5):389-99.

27 Bellamy N, et al. Recommendations for a core set of outcome measures for future phase III clinical trials in knee, hip, and hand osteoarthritis. Consensus development at OMERACT III. J Rheumatol. 1997;24(4):799-802.

28 Higgins JPT, Green S (editors). Cochrane Handbook for Systematic Reviews of Interventions Version 5.1.0 [updated March 2011]. The Cochrane Collaboration, 2011. Available from www.handbook.cochrane.org.

29 Tubach F, et al. Minimum clinically important improvement and patient acceptable symptom state in pain and function in rheumatoid arthritis, ankylosing spondylitis, chronic back pain, hand osteoarthritis, and hip and knee osteoarthritis: results from a prospective multinational study. Arthritis Care Res. 2012;64(11):1699-707.

30 Wandel S, et al. Effects of glucosamine, chondroitin, or placebo in patients with osteoarthritis of hip or knee: network meta-analysis. BMJ. 2010;341:c4675.

31 Donders ART, et al. Review: a gentle introduction to imputation of missing values. J Clin Epidemiol. 2006;59(10):1087-91.

32 Koopman L, et al. Comparison of methods of handling missing data in individual patient data meta-analyses: an empirical example on antibiotics in children with acute otitis media. Am J Epidemiol. 2008;167(5):540-5.

33 Debray $T$, et al. Get real in individual participant data (IPD) meta-analysis: a review of the methodology. Res Synth Methods. 2015;6(4):293-309.

34 Higgins JP, et al. Measuring inconsistency in meta-analyses. BMJ. 2003; 327(7414):557.

35 Evangelou E, loannidis JP, Patsopoulos NA. Uncertainty in heterogeneity estimates in meta-analyses. BMJ. 2007;335(7626):914-6.

36 Sterne JA, Gavaghan D, Egger M. Publication and related bias in metaanalysis: power of statistical tests and prevalence in the literature. J Clin Epidemiol. 2000;53(11):1119-29.

37 Begg CB, Berlin JA. Publication bias: a problem in interpreting medical data. J R Stat Soc A Stat Soc. 1988:419-63.

38 Reginster J-Y, et al. Recommendations for an update of the 2010 European regulatory guideline on clinical investigation of medicinal products used in the treatment of osteoarthritis and reflections about related clinically relevant outcomes: expert consensus statement. Osteoarthr Cartil. 2015; 23(12):2086-93.

39 Riley RD, Lambert PC, Abo-Zaid G. Meta-analysis of individual participant data: rationale, conduct, and reporting. BMJ. 2010;340:C221.

40 Stewart LA, Tierney JF. To IPD or not to IPD? Advantages and disadvantages of systematic reviews using individual patient data. Eval Health Prof. 2002; 25(1):76-97.

41 Clarke MJ, Stewart LA. Obtaining individual patient data from randomised controlled trials, Systematic Reviews in Health Care: Meta-Analysis in Context, Second Edition. 2001. p. 109-21.

\section{Submit your next manuscript to BioMed Central and we will help you at every step:}

- We accept pre-submission inquiries

- Our selector tool helps you to find the most relevant journal

- We provide round the clock customer support

- Convenient online submission

- Thorough peer review

- Inclusion in PubMed and all major indexing services

- Maximum visibility for your research

Submit your manuscript at www.biomedcentral.com/submit 